\title{
Research on the Pragmatic Function of Vague Language in English TV Interview
}

\author{
Fu Bo \\ College of Foreign Languages, Jilin Agricultural University, XinCheng Street, Changchun, China \\ ivy510@126.com
}

Keywords: Vague Language; English TV interview; Cooperative Principle(CP); Politeness Principle(PP); Pragmatic Function

\begin{abstract}
It is believed that in our daily communication, speakers should use accurate language and avoid unclear and vague expression. However, in practice, because of the objective existence of vague phenomenon and certain communicative purposes, both parties of the communication will use vague language consciously. In the recent years, the wide employment of vague language in communication has evoked increasing interest at home and aboard. The relevant literature reveals that many scholars have studied vague language in different contexts, but fewer have tried to carry through study on vagueness in English TV interview from the pragmatic perspective. Because of this fact, the author tries to be devoted to the systemic analysis of vagueness and its pragmatic functions in English TV interviews. The thesis aims at studying vague language from the perspective of pragmatics under the guidance of H.P. Grice's Cooperative Principle (CP) and Geoffrey Leech's Politeness Principle (PP). The English texts from the recent CCTV-9, VOA, BBC, CNN's TV interviews will be chosen as the research materials, and the key points to be investigated are as follows:1. Why do the interviewer and interviewee have to use vague language? 2. How do the both parties in English TV Interviews maneuver the CP and PP through vagueness to realize their intended meaning? What are the major devices? 3.What are the pragmatic functions of vague language in English TV interviews? The analysis of vagueness in English TV Interviews attempts to improve our understanding of the language in English TV Interviews and the communicative purposes of participants and help us to interpret English TV Interviews in a better way. In the meanwhile, it is hoped that the present study could offer some inspiration to language teaching, especially foreign language teaching, which advocates the clear and precise expression.
\end{abstract}

\section{Introduction}

"Vague phenomenon exists in natural world and vague language permeates our daily communication.” by Channel in 1994. Though many people hold that the words and expressions arousing vagueness and ambiguity should be avoided, in our practice of using language, we realize that sometimes people express indirectly with vague expression consciously or unconsciously. His viewpoint shows that vagueness in communication is part of our taken-for-granted world, and that normally we do not notice it unless it appears inappropriate--for example, when someone seems to be deliberately withholding information.

There is a circumstance in which we are bound employ vague language. When we do not know the exact name of a flower, "jasmine", "rose" or "lily", we could employ the vague word "flower" to refer to it. From this case, we could know that the employment of vague language is the deliberate activity with certain communicative intentions.

Vague language exists in nearly all the language use. However, the current pragmatic researches on vagueness are mostly based on the formal, written discourse or daily conversation. Comparatively speaking, there are few academic books and papers on the mixture, for example, TV Interviews, where lots of vague language are also used in certain situations and play an important role. As a special communicative platform, interview is a kind of informative and on-the-spot activity. In order to convey the information correctly and effectively without careful preparation, 
and build a positive image before the public, both parties in the interview have to employ vague language in their utterance. The utilization of vague language is deliberate, and there are some communicative intentions behind, which deserve our study from the perspective of pragmatics.

The research significance of this thesis is as follows: Theoretically speaking, the application of pragmatic theories to study the vagueness in English TV Interviews can broaden the scope of the study on vagueness and can provide a new perspective for the linguistic study of English TV Interview. Practically speaking, it is of some help for the other part of the interview and audience to have a better understanding of the motivation of the utterance and the information the speaker conveys. Furthermore, it is also of some value to the language teaching, especially the foreign language teaching which advocates the precise expression.

\section{Concepts of English TV Interview and Vagueness}

Nowadays, media is penetrating into our daily lives in every aspect. TV interview has been an integral part of mass media all over the world, and then aroused great attention from many scholars in linguistics, anthropology, culture and psychology. The history of TV interview could be traced back to 1950s, when the first TV interview appeared in American media, which was called "talk show". While in China, the appearance of TV interviews is comparatively later than other countries. Since the implementation of Reform and Opening-up Policy in 1980s, China has undergone dramatic changes. One of the greatest changes is the globalization of medium, which has brought the English programs into China.

Peirce is universally recognized as the originator of the concept of vagueness and formulates it in a rigorous way. According to Peirce's definition, speakers are allowed to produce utterances without having decided whether certain facts are "excluded or allowed by" them.

\section{Application of Cooperative Principle and Politeness Principle to Vague Language}

In 1796, H.P. Grice formulates the Cooperative Principle as follows: Make your conversational contribution such as is required at the stage at which it occurs, by the accepted purpose or direction of the talk exchange in which you are engaged. Grice classifies his principle into quality maxim,four categories which he calls maxims namely quantity maxim relation maxim and manner maxim.Grice' Cooperative Principle and his maxims are what participants have to observe in order to communicate in an efficient way. That means that the speakers should speak sincerely, relatively, and clearly and provide sufficient information. He argues that as long as participants exchange in conversation, each of them will assume that the other party is adhering to the Cooperative Principle. However, he also points out that these maxims are not always observed, and sometimes people will violate deliberately them in conversation. Generally speaking, people involved in the communication set up a cooperative relationship, and assume others abide by CP. When the speaker violates one maxim or several maxims, conversational implicature is generated. However, Grice's $\mathrm{CP}$ is not complete, which does not reveal the reasons why speakers will deliberately violate these maxims. Later, based on Cooperative Principle, Politeness Principle has been proposed by Geoffrey Leech to solve this problem.

In 1983, Leech in his book Principle of Pragmatics defined politeness as forms of behavior that establish and maintain the social community and proposed his "Politeness Principle" as a way trying to "rescue" CP and answer the following two questions: Why people are often so indirect in conveying what they mean; and what is the relation between sense and force when non-declarative types of sentences are being considered. Generally speaking, Cooperative Principle and Politeness Principle are complementary to each other during our daily communication and act as a general wide to language use.

Generally speaking, there are two kinds of vague devices. The first one is called hedge. After being proposed by George Lakoff to describe "the word acting to make things fuzzier or less fuzzy", hedge has subsequently been applied as a linguistic device to qualify a speaker's confidence in the truth of a proposition. In present study, in order to explore the pragmatic values of hedge in English 
TV Interviews, the classification of hedge in present study is primarily based on the communicative modal of CP and PP.

The other one is vague word, which refers to the word with the vague nature. Most of the vocabularies in natural language, especially those words concerned with such concepts as time, space, range, temperament, status, change, sentiment, etc. have indeterminacy in terms of their meaning items whose vagueness can be reflected in nouns, verbs, pronouns, adjectives, adverbs and conjunctions. Such words as "morning”, “afternoon”, “elder”, “young”, "here” etc. are indeterminate in terms of extension. However, the core meanings of vague words are determinate, which guarantees the conveyance of information in an accurate way. In English TV interviews, to produce vagueness in utterance through vague words can also help the interviewer and interviewee well cope with some tough issues when necessary.

Through the careful analysis of the collected materials, the author finds out that hedges and vague words achieve their application in English TV interviews through different measures. Hedges work by making the related proposition fuzzier or less fuzzy while vague words work by the vague meaning conveyed by themselves. The following is the detailed discussion about how the two devices work under the guidance of CP and PP.

In order to achieve communicative goals, the first device the speakers would like to choose is hedge, which could make the proposition fuzzier or less fuzzy. Hedge is composed of two categories: CP hedge and PP hedge. There is infinite phenomenon in the world which defies the precise description by finite word. In English TV interviews, to produce vagueness in utterance by using vague words can help the interviewer and interviewee well cope with some tough issues when necessary. For this category includes such a big scope, the author will mainly select five pieces of interviews, including politics, economy, culture, technology and entertainment, to illustrate how the vague words are employed in English TV interviews.

Hedge could change the truth-fact of the proposition and make the utterance vaguer, while vague words could provide a vague utterance through the vague meaning conveyed by themselves. Under the guidance of Cooperative Principle and Politeness Principle, hedge could be classified into CP Hedge and PP Hedge, each of which could be further divided into several sub-categories according to the maxims they abide by or violate. Furthermore, the two devices are not independent, but could be used in a combined way. Through the analysis of typical examples, it is found that utilization of $\mathrm{CP}$ hedge, PP hedge and vague words are all the requirement of smooth communication.

\section{The Pragmatic Function of Vague Language in English TV Interview}

Just as Brown\&Yule point out, what the speaker or writer has primarily in mind is the efficient transference of information. However, social communication is a complicated process. Although the major function of vague language is to transfer more information, many other functions can be detected. Through typical examples, the author draws a conclusion that vague language is employed in English TV interview mainly to achieve the following pragmatic functions. (1) Giving the right amount of information; (2) Increasing the flexibility and self-protecting; (3) Achieving politeness; (4) Creating harmonious atmosphere; (5) Being tactful and changing topic. These five functions are of interaction, that is, the utilization of vague language may realize the five pragmatic purposes respectively or simultaneously. Moreover, the author also points out that ostensible use of vague language should be avoided and offers some suggestions for the proper employment of it.

\section{Summary}

Vagueness is the intrinsic attribute of language and vague language is ubiquitous and unavoidable in communication. Hence, it is impossible for people to get along with a language in which all terms were definite. The employment of vague language helps both parties of communication to achieve certain communicative purpose. To explore how vague language achieves its application in English Interviews, the thesis takes Grice's Cooperative Principle and Leech's Politeness Principle as the guiding theory to analyze the major vague devices and their 
pragmatic functions. After a comparatively systematic and detailed analysis, the thesis comes to the following conclusions. To begin with, vagueness prevails in English TV interviews, and manifest itself mainly in observation or deliberately violation of maxims of Cooperative Principle and Politeness Principe separately or simultaneously. Cooperative Principle is the basic premise the speakers have to follow in communication, while Politeness Principle regulates speakers to convey their information in a harmonious atmosphere. The two principles are combined to guide the use of vague language in English TV Interviews.

In the second place, both parties of the interview manage their statements by using two linguistic devices, namely hedge and vague words to achieve their communicative purposes. Hedge includes CP hedge and PP hedge, each of which could be further divided into several sub-categories according to the maxims they abide by or violate. And vague words include vague nouns, verbs, pronouns, adjectives, adverbs and conjunctions. In addition; vague language as a communicative tool, has its special pragmatic functions in English TV interviews: (1) Giving the right amount of information; (2) Improving the flexibility of language and self-protecting; (3) Achieving politeness; (4) Creating harmonious atmosphere; (5) Being tactful and changing topic. These five functions are interactional, that is, they may work at the same time or respectively. Last but not the least; the thesis also suggests that abusing vague language should be avoided. Proper employment of vague language in English TV interviews and daily conversation is helpful to reveal the truth of the events, convey the positive information and build good social image.

\section{References}

[1] Babak Ghasemi, Masoud Hashemi. ICT: A Pragmatic Study of Mitigation in Television Interview Talks (2011)

[2] Cappelli, Gregory, Scott Wilson, Michael Husman. Principles of Pragmatics (2010)

[3] Loanne Snavely, Helen Smith. ACRL Eleventh National Conference, Bringing the Library to Students: Linking Customized Library Resources through a Course- Management System (2003)

[4] Kong K. Vagueness form a Pragmatic Perspective (2009)

[5] Zadeh. L.A. Sets and Fuzzy Information Granulation Theory Foreign Language Teaching and Research Press. (2007)

[6] Jiang Shujuan. A Study of Evasive Answer in Political Interviews from the Perspective of Cognitive Pragmatics (2007)

[7]Shen Yongsheng. Basic Acts Analysis of Host Discourse of the TV Talk Show Program University Press (2004) 